\title{
Analytical Solution of the Hyperbolic Heat Conduction Equation for Moving Semi-Infinite Medium under the Effect of Time-Dependent Laser Heat Source
}

\author{
R. T. Al-Khairy and Z. M. AL-Ofey \\ Department of Mathematics, King Faisal University, P.O. Box 1982, Dammam 31413, Saudi Arabia
}

Correspondence should be addressed to Z. M. AL-Ofey, zakiah-alofey@hotmail.com

Received 28 June 2008; Revised 2 March 2009; Accepted 12 May 2009

Recommended by George Jaiani

\begin{abstract}
This paper presents an analytical solution of the hyperbolic heat conduction equation for moving semi-infinite medium under the effect of time dependent laser heat source. Laser heating is modeled as an internal heat source, whose capacity is given by $g(x, t)=I(t)(1-R) \mu e^{-\mu x}$ while the semi-infinite body has insulated boundary. The solution is obtained by Laplace transforms method, and the discussion of solutions for different time characteristics of heat sources capacity (constant, instantaneous, and exponential) is presented. The effect of absorption coefficients on the temperature profiles is examined in detail. It is found that the closed form solution derived from the present study reduces to the previously obtained analytical solution when the medium velocity is set to zero in the closed form solution.
\end{abstract}

Copyright (c) 2009 R. T. Al-Khairy and Z. M. AL-Ofey. This is an open access article distributed under the Creative Commons Attribution License, which permits unrestricted use, distribution, and reproduction in any medium, provided the original work is properly cited.

\section{Introduction}

An increasing interest has arisen recently in the use of heat sources such as lasers and microwaves, which have found numerous applications related to material processing (e.g., surface annealing, welding and drilling of metals, and sintering of ceramics) and scientific research (e.g., measuring physical properties of thin films, exhibiting microscopic heat transport dynamics). Lasers are also routinely used in medicine. In literature, many researchers have investigated the heat transfer for moving medium under the effect of the classical Fourier heat conduction model [1,3-6].

In applications involving high heating rates induced by a short-pulse laser, the typical response time is in the order of picoseconds [7-10]. In such application, the classical Fourier heat conduction model fails, and the use of Cattaneo-Vernotte constitution is essential [11, 12]. 
In this constitution, it is assumed that there is a phaselag between the heat flux vector $(\underline{q})$ and the temperature gradient $(\underline{\nabla} T)$. As a result, this constitution is given as

$$
\underline{q}+\tau \frac{\partial \underline{q}}{\partial t}=-\kappa \underline{\nabla} T
$$

where $\kappa$ is the thermal conductivity and $\tau$ is the relaxation time (phase lag in heat flux). The energy equation under this constitution is written as

$$
\rho C_{p} \tau \frac{\partial^{2} T}{\partial t^{2}}+\rho C_{p} \frac{\partial T}{\partial t}=\kappa \nabla^{2} T+\left(\tau \frac{\partial g}{\partial t}+g\right)
$$

In the literature, numerous works have been conducted using the microscopic hyperbolic heat conduction model [10,13-18]. To the authors' knowledge, the thermal behavior of moving semi-infinite medium subject to Time-Dependent laser heat source, under the effect of the hyperbolic heat conduction model, has not been investigated yet. In the present work, the thermal behavior of moving semi-infinite medium subject to TimeDependent laser heat source, under the effect of the hyperbolic heat conduction model, is investigated.

\section{Mathematical Model}

In this paper heat distribution in a moving semi-infinite medium due to internal laser heat source is considered. Our medium at $t=0$ is occupying the region $x \geq 0$ with insulated surface at $x=0$. Moreover, at time $t=0$, the temperature field within the medium is uniform with a value $T_{0}$ and stationary.

We consider first a semi-infinite medium moving with a constant velocity $u$ in the direction of the $x$-axis, if heat generation is present within the material, the balance law for the internal energy can be expressed in terms of $T$ as

$$
\rho C_{p} \frac{D T}{D t}+\frac{\partial q}{\partial x}=g(x, t),
$$

where

$$
\frac{D}{D t} \equiv \frac{\partial}{\partial t}+u \frac{\partial}{\partial x}
$$

which denotes the material derivative.

If the body is in motion, the Maxwell-Cattaneo law (1.1) leads to a paradoxical result so that by replacing the partial time derivative in (1.1) with the material derivative operator, the paradox is removed, and the material form of the Maxwell-Cattaneo law is strictly Galilean invariant. Therefore, (1.1) is replaced by [19]

$$
q+\tau\left(\frac{\partial q}{\partial t}+u \frac{\partial q}{\partial x}\right)=-\kappa \frac{\partial T}{\partial x}
$$


Elimination of $q$ between (2.1) and (2.3) yields the heat transport equation

$$
\tau \frac{\partial^{2} T}{\partial t^{2}}+\frac{\partial T}{\partial t}+u \frac{\partial T}{\partial x}+2 \tau u \frac{\partial^{2} T}{\partial x \partial t}+\tau\left(u^{2}-c^{2}\right) \frac{\partial^{2} T}{\partial x^{2}}=\frac{1}{\rho C_{p}}\left[g+\tau \frac{\partial g}{\partial t}+\tau u \frac{\partial g}{\partial x}\right]
$$

where the initial and boundary conditions are given by

$$
\begin{aligned}
& T(x, 0)=T_{0},\left.\quad \frac{\partial T}{\partial t}\right|_{t=0}=\frac{g}{\rho C_{p}}, \quad x \geq 0 \\
& \frac{\partial T}{\partial x}(0, t) c=0, \quad \frac{\partial T}{\partial x}(\infty, t)=0, \quad t>0 .
\end{aligned}
$$

The relaxation time is related to the speed of propagation of thermal wave in the medium, $c$, by

$$
\tau=\frac{\alpha}{c^{2}}
$$

The heat source term in (2.4) which describes the absorption of laser radiation is modeled as [20]

$$
g(x, t)=I(t)(1-R) \mu \exp (-\mu x)
$$

where $I(t)$ is the laser incident intensity, $R$ is the surface reflectance of the body, and $\mu$ is the absorption coefficient.

We consider semi-infinite domains, which have initial temperature equal to the ambient one. The following dimensionless variables are defined:

$$
X=\frac{x}{2 c \tau}, \quad \eta=\frac{t}{2 \tau}, \quad \theta=\frac{\left(T-T_{0}\right)}{\left(T_{m}-T_{0}\right)}, \quad U=\frac{u}{c}, \quad S=\frac{\tau g}{\rho C_{p}\left(T_{m}-T_{0}\right)} .
$$

Equation (2.4) is expressed in terms of the dimensionless variables (2.9) as

$$
2 \frac{\partial \theta}{\partial \eta}+2 U \frac{\partial \theta}{\partial X}+\frac{\partial^{2} \theta}{\partial \eta^{2}}+2 U \frac{\partial^{2} \theta}{\partial \eta \partial X}-\left(1-U^{2}\right) \frac{\partial^{2} \theta}{\partial X^{2}}=\left[4 S+2 \frac{\partial S}{\partial \eta}+2 U \frac{\partial S}{\partial X}\right] .
$$

The dimensionless heat source capacity according to (2.8) is

$$
S=\psi_{0} \phi(\eta) \exp (-\beta X)
$$

where

$$
\psi_{0}=\frac{\tau I_{r}(1-R) \mu}{\rho C_{p} T_{0}}, \quad \phi(\eta)=\frac{I(2 \tau \eta)}{I_{r}}, \quad \beta=2 c \tau \mu
$$


The dimensionless initial conditions for the present problem are

$$
\begin{gathered}
\theta(X, 0)=0, \\
\frac{\partial \theta}{\partial \eta}(X, 0)=2 \psi_{0} \phi(0) \exp (-\beta X) .
\end{gathered}
$$

The results from the assumption are that there is no heat flow in the body at the initial moment [21], that is,

$$
q(X, 0)=0
$$

The dimensionless boundary conditions are

$$
\begin{gathered}
\frac{\partial \theta}{\partial X}(0, \eta)=0, \\
\frac{\partial \theta}{\partial X}(\infty, \eta)=0, \quad \eta>0 .
\end{gathered}
$$

We substitute (2.11) for $S$ in (2.10) to obtain

$$
2 \frac{\partial \theta}{\partial \eta}+2 U \frac{\partial \theta}{\partial X}+\frac{\partial^{2} \theta}{\partial \eta^{2}}+2 U \frac{\partial^{2} \theta}{\partial X \partial \eta}-\left(1-U^{2}\right) \frac{\partial^{2} \theta}{\partial X^{2}}=2 \psi_{0}\left[(2-U \beta) \phi(\eta)+\frac{\partial \phi}{\partial \eta}\right] \exp (-\beta X) .
$$

\section{Analytical Solution}

Taking the Laplace transform of (2.18), using the initial conditions given by (2.13) and (2.14), yields

$$
\left(1-U^{2}\right) \frac{\partial^{2} \bar{\theta}}{\partial X^{2}}-2 U(1+s) \frac{\partial \bar{\theta}}{\partial X}-s(2+s) \bar{\theta}=-2 \psi_{0}(2+s-U \beta) \bar{\phi} \exp (-\beta X)
$$

where

$$
\begin{aligned}
\bar{\theta}(X, s) & =L[\theta(X, \eta)] \\
\bar{\phi}(s) & =L[\phi(\eta)] .
\end{aligned}
$$

The transformed boundary conditions given by (2.16) and (2.17) are

$$
\begin{gathered}
\frac{d \bar{\theta}}{d X}(0, s)=0 \\
\frac{d \bar{\theta}}{d X}(\infty, s)=0 .
\end{gathered}
$$


Journal of Applied Mathematics

Equation (3.1) has homogeneous $\left(\overline{\theta_{h}}\right)$ and particular $\left(\overline{\theta_{p}}\right)$ solutions. Therefore, $\bar{\theta}$ yields

$$
\bar{\theta}=\overline{\theta_{h}}+\overline{\theta_{p}}
$$

The mathematical arrangement of the solution of (3.1) is given in Appendix A. Consequently, (3.1) for $X>0$ yields

$$
\theta(X, \eta)=\left\{\begin{array}{l}
2 \psi_{0} \exp (-\beta X) f(\eta), \quad \text { for } \eta \leq \frac{X}{1+U^{\prime}}, \\
2 \psi_{0} \exp (-\beta X) f(\eta)-2 \beta \psi_{0} \int_{X / 1+U}^{\eta} \exp (-y) I_{0}\left(\sqrt{a} \sqrt{\left(y+\frac{U X}{a}\right)^{2}-\frac{X^{2}}{a^{2}}}\right) \\
\times h_{8}(\eta-y) d y-2 \beta \psi_{0}(1+U) \int_{X / 1+U}^{\eta} \exp (-y) I_{0}\left(\sqrt{a} \sqrt{\left(y+\frac{U X}{a}\right)^{2}-\frac{X^{2}}{a^{2}}}\right) \\
\quad \times h_{7}(\eta-y) d y, \text { for } \eta>\frac{X}{1+U^{\prime}},
\end{array}\right.
$$

where

$$
\begin{gathered}
0<U<1, \\
f(\eta)=\frac{1}{2 \gamma} \int_{0}^{\eta} \phi(r)\left\{\left(\gamma_{p}-U \beta\right) \exp \left[\gamma_{m}(\eta-r)\right]+\left(\gamma_{m}+U \beta\right) \exp \left[-\gamma_{p}(\eta-r)\right]\right\} d r, \\
h_{7}(\eta)=f(\eta)+U^{2} \int_{0}^{\eta} \phi(r)\left[D_{1} \exp (-2(\eta-r))+D_{2}\right. \\
\left.+D_{3} \exp \left(\gamma_{m}(\eta-r)\right)+D_{4} \exp \left(-\gamma_{p}(\eta-r)\right)\right] d r, \\
h_{8}(\eta)=U \sqrt{a} \int_{0}^{\eta} \exp (-v) I_{1}(\sqrt{a} v) h_{7}(\eta-v) d v, \\
\gamma=\sqrt{1+\beta^{2}}, \\
\gamma_{m}=\gamma-(1-U \beta), \\
\gamma_{p}=\gamma+(1-U \beta), \\
\frac{-U \beta}{2\left(2+\gamma_{m}\right)\left(-2+\gamma_{p}\right)}, \quad D_{2}=\frac{-2+U \beta}{2 \gamma_{m} \gamma_{p}}, \\
\frac{(\gamma+1)}{2 \gamma \gamma_{m}\left(2+\gamma_{m}\right)}, \quad D_{4}=\frac{(\gamma-1)}{2 \gamma \gamma_{p}\left(-2+\gamma_{p}\right)} .
\end{gathered}
$$




\section{Solutions for Special Cases of Heat Source Capacity}

The temperature distributions resulting from any specified time characteristics of the heat source $\phi(\eta)$ are available using the general hyperbolic solution (3.7)-(3.14). However, for some particular $\phi(\eta)$ the general solution can be considerably simplified. Some of such cases are discussed below.

\subsection{Source of Constant Strength: $\phi(\eta)=1$}

This case may serve as a model of a continuously operated laser source. It may be also used as a model of a long duration laser pulse when the short times (of the order of few or tens $\tau$ ) are considered. For $\phi(\eta)=1,(3.9)$ and (3.10) are reduced, respectively, to

$$
\begin{gathered}
f_{1}(\eta)=\frac{\gamma_{p}\left(\gamma_{p}+U \beta\right) \exp \left(\gamma_{m} \eta\right)-\gamma_{m}\left(\gamma_{m}-U \beta\right) \exp \left(-\gamma_{p} \eta\right)-2 \gamma(2-U \beta)}{2 \gamma \gamma_{m} \gamma_{p}}, \\
h_{7(1)}(\eta)=f_{1}(\eta)+U^{2}\left\{D_{1} \exp (-\eta) \sinh (\eta)+D_{2} \eta+\frac{D_{3}}{\gamma_{m}}\left[\exp \left(\gamma_{m} \eta\right)-1\right]\right. \\
\left.+\frac{D_{4}}{\gamma_{p}}\left[1-\exp \left(-\gamma_{p} \eta\right)\right]\right\} .
\end{gathered}
$$

4.2. Instantaneous Source: $\phi(\eta)=\delta(\eta)$

In this case, (3.9) and (3.10) take the form, respectively,

$$
\begin{gathered}
f_{2}(\eta)=\frac{1}{2 \gamma}\left[\left(\gamma_{p}+U \beta\right) \exp \left(\gamma_{m} \eta\right)+\left(\gamma_{m}-U \beta\right) \exp \left(-\gamma_{p} \eta\right)\right] \\
h_{7(2)}(\eta)=f_{2}(\eta)+U^{2}\left[D_{1} \exp (-2 \eta)+D_{2}+D_{3} \exp \left(\gamma_{m} \eta\right)+D_{4} \exp \left(-\gamma_{P} \eta\right)\right]
\end{gathered}
$$

\subsection{Exponential Source: $\phi(\eta)=\exp (-v \eta)$}

In this case (3.9) and (3.10) are as follows, respectively,

$$
\begin{gathered}
f_{3}(\eta)=\frac{1}{2 \gamma\left(v-\gamma_{p}\right)\left(v+\gamma_{m}\right)}\left[\left(v+\gamma_{m}\right)\left(\gamma_{m}-U \beta\right) \exp \left(-\gamma_{p} \eta\right)+\left(v-\gamma_{p}\right)\left(\gamma_{p}+U \beta\right) \exp \left(\gamma_{m} \eta\right)\right. \\
+2 \gamma(2-v-U \beta) \exp (-v \eta)], \\
h_{7(3)}(\eta)=f_{3}(\eta)+U^{2}\left\{\frac{D_{1}}{(-2+v)} \exp (-2 \eta)+\frac{D_{2}}{v}+\frac{D_{3}}{\left(v+\gamma_{m}\right)} \exp \left(\gamma_{m} \eta\right)\right. \\
\left.+\frac{D_{4}}{\left(v-\gamma_{p}\right)} \exp \left(-\gamma_{p} \eta\right)+D_{5} \exp (-v \eta)\right\},
\end{gathered}
$$




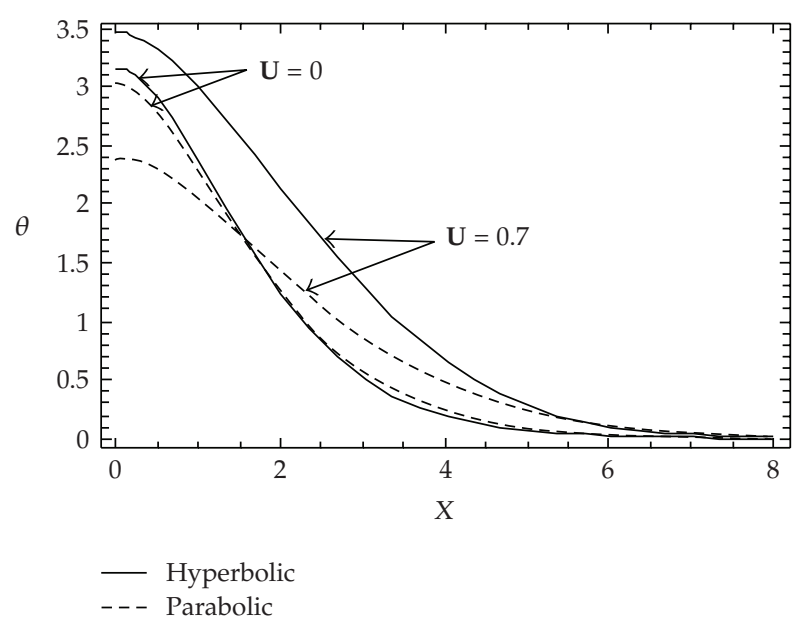

Figure 1: Dimensionless temperature distributions resulting from the hyperbolic and parabolic models with dimensionless velocity of the medium for the heat source of constant strength; $\phi(\eta)=1, \psi_{0}=$ 1 , and $\beta=1, \eta=3$.

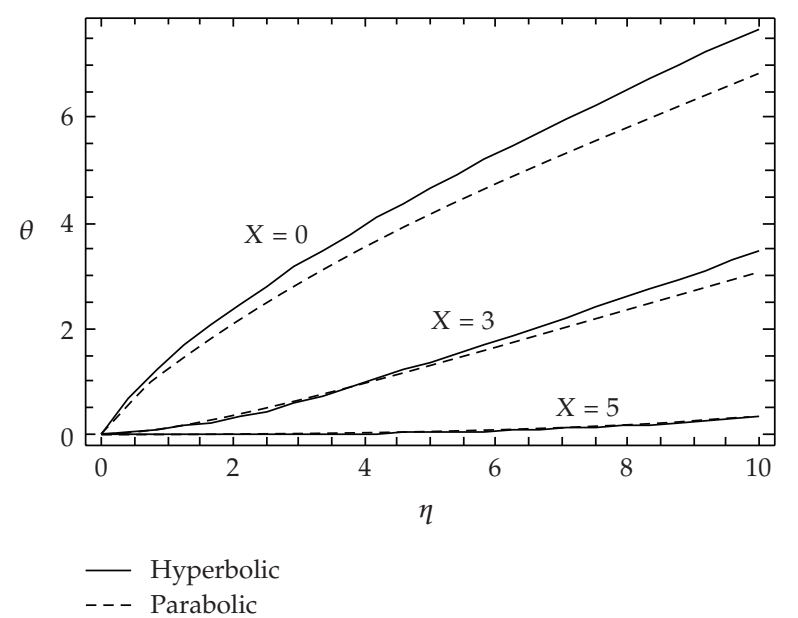

Figure 2: Variation of dimensionless temperature with dimensionless time at different points of the body for the heat source of constant strength; $\phi(\eta)=1, \psi_{0}=1$, and $U=0.1$.

where

$$
D_{5}=\frac{D_{1}}{(2-v)}-\frac{D_{2}}{v}-\frac{D_{3}}{\left(v+\gamma_{m}\right)}-\frac{D_{4}}{\left(v-\gamma_{p}\right)}
$$

\section{Results and Discussion}

Using the solutions for arbitrary $\phi(\eta)$ and the solutions for the special cases we calculated, with the aid of the program Mathematica 5.0, and we performed calculations for metals putting $\psi_{0}=1$ and $\beta=0.5$ or 1 , since we assumed that typical values of the model parameters 


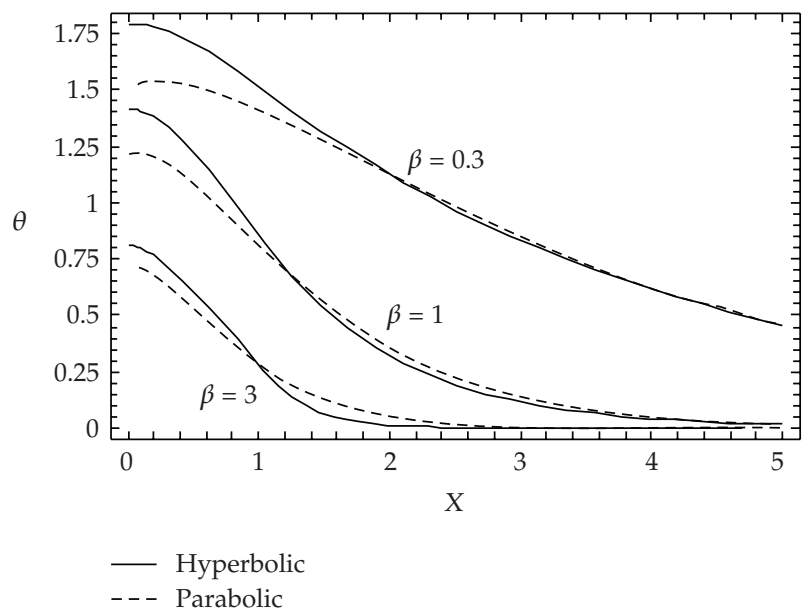

Figure 3: Dimensionless temperature distributions at $\eta=1$ for the heat source of constant strength and various values of $\beta ; \phi(\eta)=1, \psi_{0}=1$, and $U=0.1$.

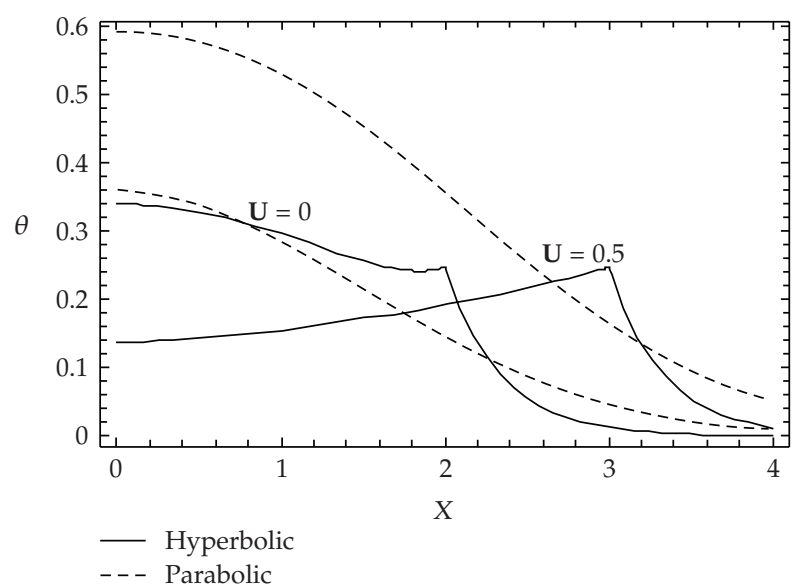

Figure 4: Dimensionless temperature distributions resulting from the hyperbolic and parabolic models with dimensionless velocity of the medium for the instantaneous heat source; $\phi(\eta)=\delta(\eta), \psi_{0}=1, \beta=$ 3 , and $\eta=2$.

for metals are: $\mu$ of the order of $10^{7}-10^{8} \mathrm{~m}^{-1}, R$ of the order of $0.9, \tau$ of the order of $10^{-13}-10^{-11} \mathrm{~s}$, and $c$ of the order of $10^{3}-10^{4} \mathrm{~m} / \mathrm{s}$ [22-25]. Some solutions for other values of $\beta$ are also presented to set off the specific features of our model. The results of calculations for various time characteristics of the heat source capacity are shown in Figures 1-9. Moreover, the velocity of the medium was assumed not to exceed the speed of heat propagation.

The hyperbolic and parabolic solutions for the heat source of constant strength $[\phi(\eta)=$ $1]$ are presented in Figures 1-3. Figure 1 shows the temperature distribution in the body for the two values of dimensionless velocity of the medium, $U=0,0.7$. Figure 2 displays the time variation of temperature at the three points of the body, $X=0,3,5$. It is clearly seen that for small $X$ the temperatures predicted by the hyperbolic model are greater than the corresponding values for the Fourier model, whereas in the region of intermediate values of $X$, the situation is just the opposite. For large $X(X \gg \eta)$ the hyperbolic and parabolic 


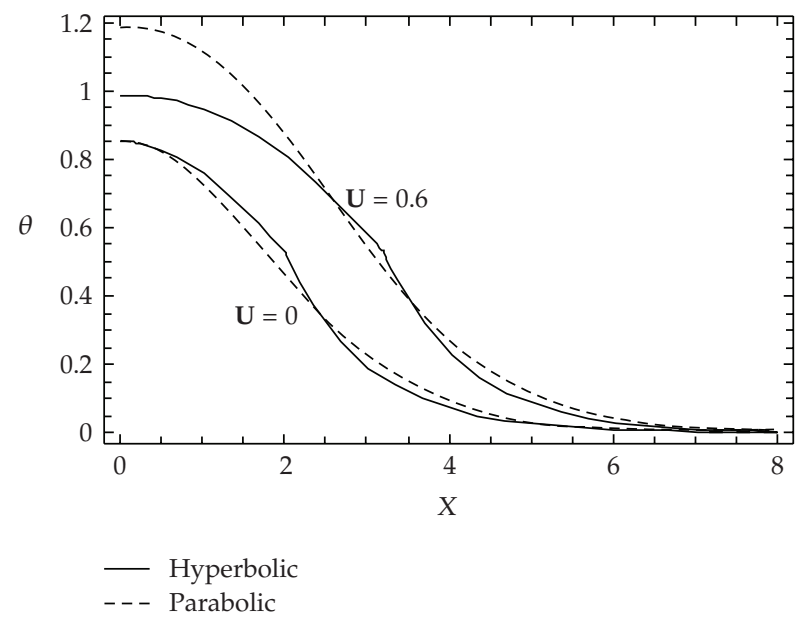

Figure 5: Dimensionless temperature distributions with dimensionless velocity of the medium for the instantaneous heat source; $\phi(\eta)=\delta(\eta), \psi_{0}=1, \beta=1$, and $\eta=2$.

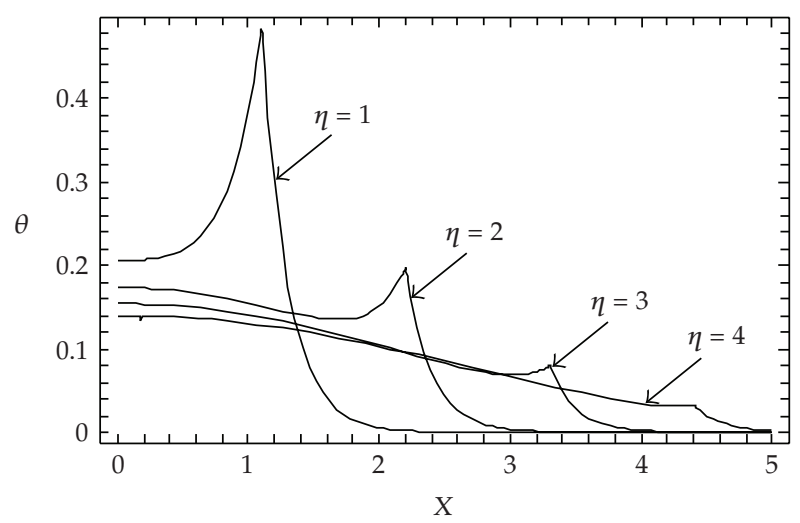

Figure 6: Dimensionless temperature distributions resulting from the hyperbolic model for the instantaneous heat source; $\phi(\eta)=\delta(\eta), \psi_{0}=1, \beta=5$, and $U=0.1$.

solutions tend to overlap. This behaviour can be explained as follows. In both models, the heat production is concentrated at the edge of the body. The same amounts of energy are generated continuously in both models, but in the case of hyperbolic models, because of the finite speed of heat conduction, more energy is concentrated at the origin of $X$ axis. This results in the higher "hyperbolic" temperature in this region and the lower in the region of intermediate $X$ values. In Figure 3, we compare the temperature distributions at $\eta=1$ resulting from the hyberbolic and parabolic for the three values of $\beta(\beta=0.3,1$, and 3). For large $\beta$, that is, when the slope of the space characteristics of the heat source capacity increases, in the hyperbolic solution, a blunt wave front can be observed. Figures $4-7$ depict the results of calculations for the instantaneous heat source $[\phi(\eta)=\delta(\eta)]$. A striking feature of the hyperbolic solutions is that the instantaneous heat source gives rise to a thermal pulse which travels along the medium and decays exponentially with time while dissipating its energy. During a period $\eta$, the maximum of the pulse moves over a distance $X=\eta(1+U)$. These effects are shown pictorially in Figures 4 and 6. Figure 4 presents the temperature 


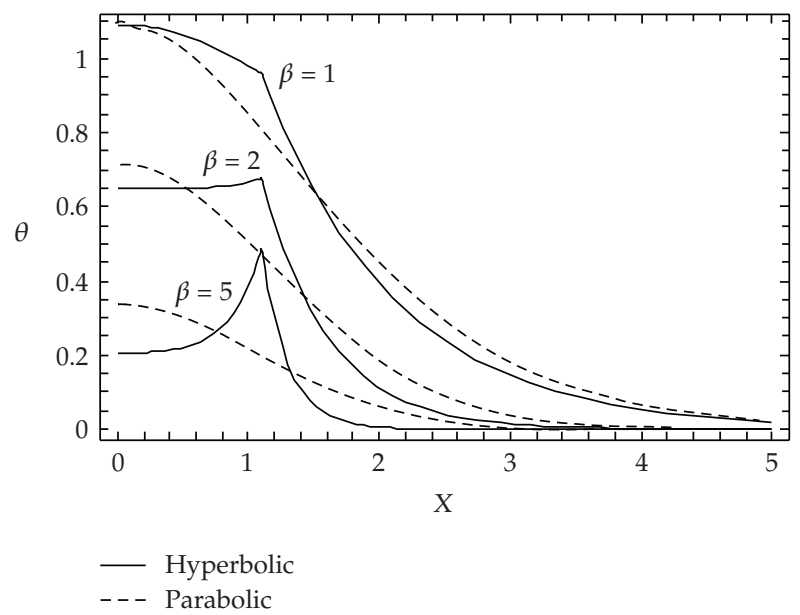

Figure 7: Dimensionless temperature distributions at $\eta=1$ for the instantaneous heat source and various values of $\beta ; \phi(\eta)=\delta(\eta), \quad \psi_{0}=1$, and $U=0.1$.

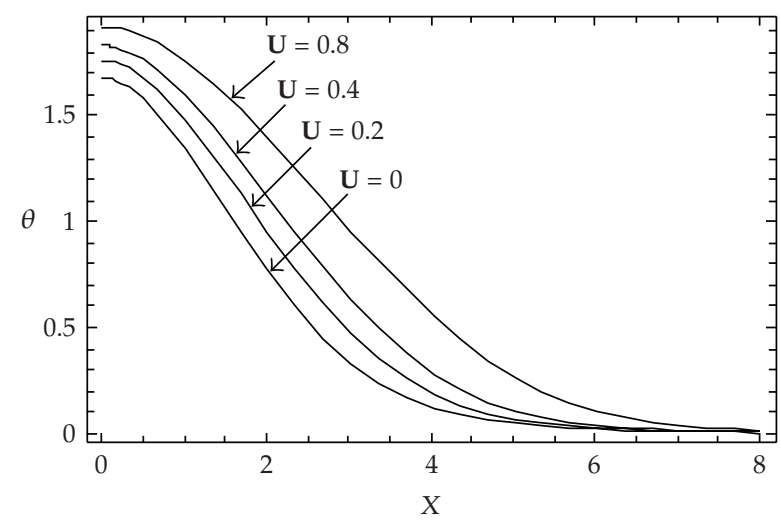

Figure 8: Dimensionless temperature distributions with dimensionless velocity of the medium from the hyperbolic model for the exponential heat source ; $\phi(\eta)=\exp (-0.4 \eta), \psi_{0}=1, \beta=1$, and $\eta=3$.

distributions in the body for $\beta=5$ and $U=0,0.5$, but Figure 6 presents the temperature distributions in the body for $\beta=5$ and $\eta=1,2,3,4$. It is seen that the pulse is not sharp but blunt exponentially, which results from the fact that in our model the heat source capacity decays exponentially along the $x$-axis. Figure 5 gives the hyperbolic and parabolic temperature distribution in the body at time $\eta=2$ for the two values of dimensionless velocity of the medium, $U=0,0.6$. Figure 7 gives the hyperbolic and parabolic temperature distribution in the body at time $\eta=1$ and velocity of the medium $U=0.1$ for various values of $\beta$. As shown in Figure 7, the smaller $\beta$ is, the more blunt the pulse and the shorter is the time of its decay is . After the decay of the pulse, the differences between the hyperbolic and parabolic solutions become only quantitative, and they vanish in short time. Figures 8 and 9 depict the results of calculations for the exponential heat source $[\phi(\eta)=\exp (-v \eta)]$. Figure 8 gives the hyperbolic temperature distribution in the body at time $\eta=3$ for the four values of dimensionless velocity of the medium, $U=0,0.2,0.4,0.8$. Figure 9 shows the temperature distribution in the body for the three values of dimensionless $\beta(\beta=0.3,1,3)$. The results are 


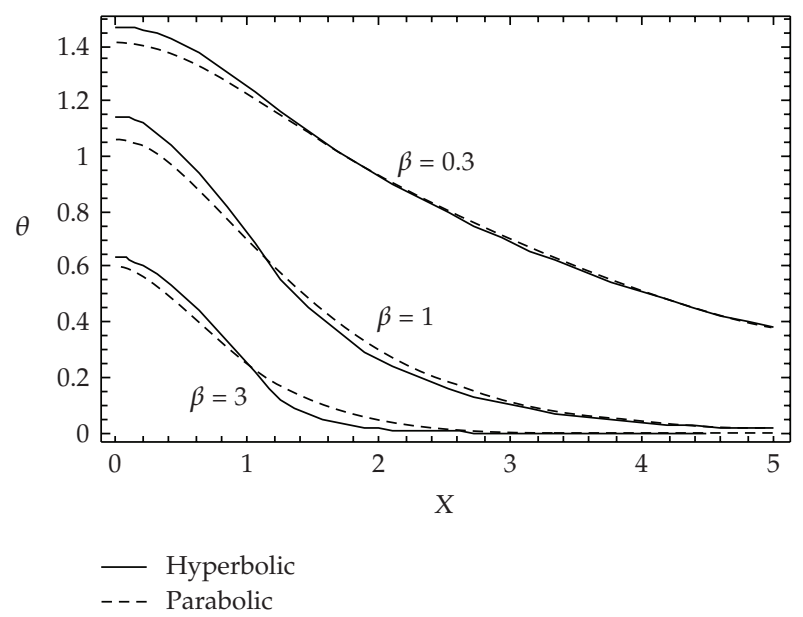

Figure 9: Dimensionless temperature distributions at $\eta=1$ for the exponential heat source and various values of $\beta ; \phi(\eta)=\exp (-0.4 \eta), \psi_{0}=1$, and $U=0.1$.

compared with those obtained from an analytical model by Lewendowska [21]. For $U=0$, our results are the same as those reported by Lewendowska [21].

\section{Conclusions}

This paper presents an analytical solution of the hyperbolic heat conduction equation for moving semi-infinit medium under the effect of Time-Dependent laser heat source. Laser heating is modeled as an internal heat source, whose capacity is given by (2.8) while the semi-infinit body was insulated boundary. The heat conduction equation together with its boundary and initial conditions have been written in a dimensionless form. By employing the Laplace transform technique, an analytical solution has been found for an arbitrary velocity of the medium variation. The temperature of the semi-infinit body is found to increase at large velocities of the medium. The results are compared with those obtained from an analytical model by Lewendowska [21]. For $U=0$, our results are the same as those reported by Lewendowska [21]. A blunt heat wavefront can be observed when the slope of the space characteristics of the heat source capacity (i.e., the value of $\beta$ ) is large.

\section{Appendix}

\section{A. Solution of Heat Transfer Equation}

The characteristic equation for the homogeneous solution can be written as

$$
r^{2}-\frac{2 U(1+s)}{1-U^{2}} r-\frac{s(2+s)}{1-U^{2}}=0
$$

which yields the solution of

$$
r_{1,2}=\frac{U(1+s)}{1-U^{2}} \pm \frac{1}{1-U^{2}} \sqrt{(1+s)^{2}-\left(1-U^{2}\right)}
$$

where $0<U<1$. 
Therefore, the homogeneous solution $\left(\overline{\theta_{h}}\right)$ yields

$$
\overline{\theta_{h}}=c_{1} \exp \left(r_{1} X\right)+c_{2} \exp \left(r_{2} X\right)
$$

or

$$
\overline{\theta_{h}}=\left[c_{1} \exp \left(\frac{U(1+s)}{a}-\frac{1}{a} \sqrt{(1+s)^{2}-a}\right) X+c_{2} \exp \left(\frac{U(1+s)}{a}+\frac{1}{a} \sqrt{(1+s)^{2}-a}\right) X\right],
$$

where $a=1-U^{2}$.

For the particular solution, one can propose $\overline{\theta_{p}}=A_{0} \exp (-\beta X)$.

Consequently, substitution of $\overline{\theta_{p}}$ into (3.1) results in

$$
\begin{aligned}
& \left(1-U^{2}\right) \beta^{2} A_{0} \exp (-\beta X)+2 U(1+s) \beta A_{0} \exp (-\beta X)-s(2+s) A_{0} \exp (-\beta X) \\
& =-2 \psi_{0}(2+s-U \beta) \bar{\phi} \exp (-\beta X)
\end{aligned}
$$

where

$$
A_{0}=\frac{-2 \psi_{0}(2+s-U \beta) \bar{\phi}}{\left[\left(1-U^{2}\right) \beta^{2}+2 U(1+s) \beta-s(2+s)\right]}
$$

or

$$
\begin{aligned}
\bar{\theta}= & c_{1} \exp \left(\frac{U(1+s)}{a}-\frac{1}{a} \sqrt{(1+s)^{2}-a}\right) X \\
& +c_{2} \exp \left(\frac{U(1+s)}{a}+\frac{1}{a} \sqrt{(1+s)^{2}-a}\right) X \\
& +\frac{2 \psi_{0}(2+s-U \beta) \bar{\phi} \exp (-\beta X)}{\left(s-\gamma_{m}\right)\left(s+\gamma_{p}\right)} .
\end{aligned}
$$

Since $\operatorname{Re}(s)>0,0<U<1$ and $d \bar{\theta} / d X(\infty, s)=0$, then $c_{2}=0$.

Therefore,

$$
\bar{\theta}=c_{1} \exp \left(\frac{U(1+s)}{a}-\frac{1}{a} \sqrt{(1+s)^{2}-a}\right) X+\frac{2 \psi_{0}(2+s-U \beta) \bar{\phi} \exp (-\beta X)}{\left(s-\gamma_{m}\right)\left(s+\gamma_{p}\right)}
$$


Journal of Applied Mathematics

By applying the boundary condition (3.4), we can obtain $c_{1}$, that is,

$$
\begin{aligned}
\frac{d \bar{\theta}}{d X}=\{ & c_{1}\left(\frac{U(1+s)}{a}-\frac{1}{a} \sqrt{(1+s)^{2}-a}\right) \times \exp \left(\frac{U(1+s)}{a}-\frac{1}{a} \sqrt{(1+s)^{2}-a}\right) X \\
& \left.-\frac{2 \beta \psi_{0}(2+s-U \beta) \bar{\phi} \exp (-\beta X)}{\left(s-\gamma_{m}\right)\left(s+\gamma_{p}\right)}\right\}_{X=0}=0,
\end{aligned}
$$

or

$$
c_{1}=\frac{2 \beta \psi_{0} a(2+s-U \beta) \bar{\phi}}{\left(U(1+s)-\sqrt{(1+s)^{2}-a}\right)\left(s-\gamma_{m}\right)\left(s+\gamma_{p}\right)} .
$$

Hence,

$$
\begin{aligned}
\bar{\theta}= & \frac{2 \beta \psi_{0} a(2+s-U \beta) \bar{\phi} \exp \left(U(1+s) / a-(1 / a) \sqrt{(1+s)^{2}-a}\right) X}{\left(U(1+s)-\sqrt{(1+s)^{2}-a}\right)\left(s-\gamma_{m}\right)\left(s+\gamma_{p}\right)} \\
& +\frac{2 \psi_{0}(2+s-U \beta) \bar{\phi} \exp (-\beta X)}{\left(s-\gamma_{m}\right)\left(s+\gamma_{p}\right)} .
\end{aligned}
$$

Let $H_{1}$ and $H_{2}$ be

$$
\begin{aligned}
H_{1}= & \frac{a(2+s-U \beta) \bar{\phi} \exp \left(U(1+s) / a-(1 / a) \sqrt{(1+s)^{2}-a}\right) X}{\left(U(1+s)-\sqrt{(1+s)^{2}-a}\right)\left(s-\gamma_{m}\right)\left(s+\gamma_{p}\right)} \\
- & {\left[\frac{\exp (-(X(1+s)) /(1+U)) \exp \left(-(X / a)\left(\sqrt{(1+s)^{2}-a}-(1+s)\right)\right)}{\sqrt{(1+s)^{2}-a}}\right.} \\
& \left.\times U \frac{\left[(1+s)-\sqrt{(1+s)^{2}-a}\right]}{\sqrt{(1+s)^{2}-a}} \times \frac{\left((1+s)^{2}-a\right)(2+s-U \beta) \bar{\phi}}{\left((1+s)^{2}-1\right)\left(s-\gamma_{m}\right)\left(s+\gamma_{p}\right)}\right]
\end{aligned}
$$




$$
\begin{aligned}
&-(1+U) {\left[\frac{\exp (-X(1+s) /(1+U)) \exp \left(-(X / a)\left(\sqrt{(1+s)^{2}-a}-(1+s)\right)\right)}{\sqrt{(1+s)^{2}-a}}\right.} \\
&\left.\times \frac{\left((1+s)^{2}-a\right)(2+s-U \beta) \bar{\phi}}{\left((1+s)^{2}-1\right)\left(s-\gamma_{m}\right)\left(s+\gamma_{p}\right)}\right] \\
&=-H_{3}-(1+U) H_{4}
\end{aligned}
$$

$$
H_{2}=\frac{(2+s-U \beta) \bar{\phi}}{\left(s-\gamma_{m}\right)\left(s+\gamma_{p}\right)}
$$

Consequently,

$$
\begin{aligned}
\theta(X, \eta) & =£^{-1} \bar{\theta}=2 \beta \psi_{0} £^{-1} H_{1}+2 \psi_{0} \exp (-\beta X) £^{-1} H_{2} \\
& =-2 \beta \psi_{0} £^{-1} H_{3}-2 \beta \psi_{0}(1+U) £^{-1} H_{4}+2 \psi_{0} \exp (-\beta X) £^{-1} H_{2} .
\end{aligned}
$$

To obtain the inverse Laplace transformation of functions $H_{2}, H_{3}$, and $H_{4}$, we use the convolution for Laplace transforms.

The Laplace inverse of $\mathrm{H}_{2}$ can be obtained as

$$
\begin{aligned}
£^{-1} H_{2} & =\frac{1}{2 \gamma} \int_{0}^{\eta} \phi(r)\left[\left(\gamma_{p}+U \beta\right) \exp \left(\gamma_{m}(\eta-r)\right)+\left(\gamma_{m}-U \beta\right) \exp \left(-\gamma_{p}(\eta-r)\right)\right] d r \\
& =f(\eta) .
\end{aligned}
$$

To obtain the inverse Laplace transformation of function $\mathrm{H}_{3}$, we use the convolution for Laplace transforms:

$$
£^{-1} H_{3}=£^{-1}\left[H_{5}(s) H_{6}(s) H_{7}(s)\right]=\int_{0}^{\eta} h_{5}(y) \int_{0}^{\eta-y} h_{6}(v) h_{7}(\eta-y-v) d v d y,
$$

where

$$
\begin{aligned}
h_{5}(\eta) & =\mathcal{E}^{-1} H_{5}=£^{-1}\left\{\frac{\exp (-(X(1+s) /(1+U))) \exp \left(-(X / a)\left(\sqrt{(1+s)^{2}-a}-(1+s)\right)\right)}{\sqrt{(1+s)^{2}-a}}\right\} \\
& =\exp (-\eta) £^{-1}\left\{\frac{\exp (-(X /(1+U)) s) \exp \left(-(X / a)\left(\sqrt{s^{2}-a}-s\right)\right)}{\sqrt{s^{2}-a}}\right\} .
\end{aligned}
$$


It is noted from the Laplace inversion that [26]

$$
\begin{gathered}
\mathcal{E}^{-1}\{H(s-b)\}=\exp (b \eta) h(\eta), \\
\mathcal{E}^{-1}\left\{\frac{\exp \left(-k\left(\sqrt{s^{2}-c^{2}}-s\right)\right)}{\sqrt{s^{2}-c^{2}}}\right\}=I_{0}\left(c \sqrt{\eta^{2}+2 k \eta}\right), \quad k \geq 0, \\
\mathcal{E}^{-1}\{\exp (-b s) H(s)\}=\left\{\begin{array}{ll}
h(\eta-b) & \text { at } \eta>b, \\
0 & \text { at } \eta<b,
\end{array} \quad b>0 .\right.
\end{gathered}
$$

Therefore,

$$
h_{5}(\eta)=£^{-1} H_{5}=\exp (-\eta) I_{0}\left(\sqrt{a} \sqrt{\left(\eta+\frac{U X}{a}\right)^{2}-\frac{X^{2}}{a^{2}}}\right), \quad \eta>\frac{X}{1+U} .
$$

Similarly, $£^{-1} H_{6}$ can be obtained, that is,

$$
\begin{aligned}
h_{6}(\eta) & =£^{-1} H_{6}=£^{-1}\left\{U \frac{\left[(1+s)-\sqrt{(1+s)^{2}-a}\right]}{\sqrt{(1+s)^{2}-a}}\right\} \\
& =U \exp (-\eta) £^{-1}\left\{\frac{\left[s-\sqrt{s^{2}-a}\right]}{\sqrt{s^{2}-a}}\right\} .
\end{aligned}
$$

It is noted from the Laplace inversion that [26]

$$
E^{-1}\left\{\frac{\left[s-\sqrt{s^{2}-c^{2}}\right]^{v}}{\sqrt{s^{2}-c^{2}}}\right\}=c^{v} I_{v}(c \eta), \quad v>-1
$$

Therefore,

$$
h_{6}(\eta)=£^{-1} H_{6}=\sqrt{a} U \exp (-\eta) I_{1}(\sqrt{a} \eta) .
$$


To obtain the inverse transformation of function $\mathrm{H}_{7}$, we use the convolution for Laplace transforms:

$$
\begin{gathered}
h_{7}(\eta)=\AA^{-1} H_{7}=\AA^{-1}\left\{\frac{\left((1+s)^{2}-a\right)(2+s-U \beta) \bar{\phi}}{\left((1+s)^{2}-1\right)\left(s-\gamma_{m}\right)\left(s+\gamma_{p}\right)}\right\} \\
=f(\eta)+U^{2} \int_{0}^{\eta} \phi(r)\left[D_{1} \exp (-2(\eta-r))+D_{2}+D_{3} \exp \left(\gamma_{m}(\eta-r)\right)\right. \\
\left.+D_{4} \exp \left(-\gamma_{p}(\eta-r)\right)\right] d r .
\end{gathered}
$$

Substituting (A.21), (A.24), and (A.25) into (A.16), it yields

$$
£^{-1} H_{3}=\int_{X /(1+U)}^{\eta} \exp (-y) I_{0}\left(\sqrt{a} \sqrt{\left(y+\frac{U X}{a}\right)^{2}-\frac{X^{2}}{a^{2}}}\right) \times h_{8}(\eta-y) d y,
$$

where

$$
h_{8}(\eta)=\int_{0}^{\eta} \sqrt{a} U \exp (-v) I_{1}(\sqrt{a} v) \times h_{7}(\eta-v) d v .
$$

Similarly, $£^{-1} \mathrm{H}_{4}$ can be obtained, after using the convolution for Laplace transforms and (A.21) and (A.25):

$$
\begin{aligned}
\mathcal{E}^{-1} H_{4} & =\mathcal{E}^{-1}\left[H_{5}(s) H_{7}(s)\right] \\
& =\int_{X /(1+U)}^{\eta} \exp (-y) I_{0}\left(\sqrt{a} \sqrt{\left(y+\frac{U X}{a}\right)^{2}-\frac{X^{2}}{a^{2}}}\right) \times h_{7}(\eta-y) d y .
\end{aligned}
$$

Substituting (A.15), (A.26), and (A.28) into (A.14), it yields

$$
\theta(X, \eta)=\left\{\begin{array}{l}
2 \psi_{0} \exp (-\beta X) f(\eta), \quad \text { for } \eta \leq \frac{X}{1+U^{\prime}}, \\
2 \psi_{0} \exp (-\beta X) f(\eta)-2 \beta \psi_{0} \int_{X /(1+U)}^{\eta} \exp (-y) I_{0}\left(\sqrt{a} \sqrt{\left(y+\frac{U X}{a}\right)^{2}-\frac{X^{2}}{a^{2}}}\right) \\
\quad \times h_{8}(\eta-y) d y-2 \beta \psi_{0}(1+U) \int_{X /(1+U)}^{\eta} \exp (-y) I_{0}\left(\sqrt{a} \sqrt{\left(y+\frac{U X}{a}\right)^{2}-\frac{X^{2}}{a^{2}}}\right) \\
\quad \times h_{7}(\eta-y) d y, \text { for } \eta>\frac{X}{1+U} .
\end{array}\right.
$$




\section{Nomenclature}

$C_{p}: \quad$ Specific heat at constant pressure, $\mathrm{J} /(\mathrm{kg} \mathrm{K})$

g: $\quad$ Capacity of internal heat source, $\mathrm{W} / \mathrm{m}^{3}$

I: $\quad$ Laser incident intensity, $\mathrm{W} / \mathrm{m}^{2}$

$I_{r}: \quad$ Arbitrary reference laser intensity

$I_{0}$ : $\quad$ Modified Bessel function, 0th order

$I_{1}$ : Modified Bessel function, 1th order

L: $\quad$ Laplace operator

$R: \quad$ Surfase reflectance

$s: \quad$ Laplace variable

$q$ : $\quad$ Heat flux vector, $\mathrm{W} / \mathrm{m}^{2}$

$\bar{t}: \quad$ Time, $\mathrm{s}$

T: $\quad$ Temperature, $\mathrm{K}$

$T_{m}, T_{0}$ : Arbitrary reference temperatures, $\mathrm{K}$

$c: \quad$ Speed of heat propagation $=(\alpha / \tau)^{1 / 2}, \mathrm{~m} / \mathrm{s}$

$x, y, z$ : Cartesian coordinates, $\mathrm{m}$

$X, Y, Z$ : Dimensionless cartesian coordinates

$S: \quad$ Dimensionless capacity of internal heat source

$u$ : Velocity of the medium, $\mathrm{m} / \mathrm{s}$

$U$ : Dimensionless velocity of the medium, $u / c$.

\section{Greek symbols}

$\alpha: \quad$ Thermal diffusivity $=\kappa /\left(\rho C_{p}\right), \mathrm{m}^{2} / \mathrm{s}$

$\kappa: \quad$ Thermal conductivity, $\mathrm{W} /(\mathrm{mK})$

$\tau: \quad$ Relaxation time of heat flux, $\mathrm{s}$

$\beta$ : $\quad$ Dimensionless absorption coefficient

$\gamma, \gamma_{m}, \gamma_{p}$ : Auxiliary coefficients defined by (3.12), (3.13), (3.14), respectively

$\phi: \quad$ Dimensionless rate of energy absorbed in the medium

$\mu: \quad$ Absorption coefficient

$\theta: \quad$ Dimensionless temperatures

$\rho: \quad$ Density

$\eta$ : $\quad$ dimensionless time

$\psi_{0}: \quad$ Internal heat source

$\bar{\theta}: \quad$ Laplace transformation of dimensionless temperature.

\section{References}

[1] J. C. Jaeger, "Moving source of heat and the temperature at sliding contacts," Proceedings of the Royal Society of NSW, vol. 76, pp. 203-224, 1942.

[2] J. C. Jaeger and H. S. Carslaw, Conduction of Heat in Solids, Oxford University Press, Oxford, UK, 1959.

[3] M. Kalyon and B. S. Yilbas, "Exact solution for time exponentially varying pulsed laser heating: convective boundary condition case," Proceedings of the Institution of Mechanical Engineers, Part C, vol. 215, no. 5, pp. 591-602, 2001.

[4] M. N. Özisik, Heat Conduction, Wiley, New York, NY, USA, 2nd edition, 1993.

[5] D. Rosenthal, "The theory of moving sources of heat and its application to metal treatments," Transaction of the American Society of Mechanical Engineers, vol. 68, pp. 849-866, 1946. 
[6] B. S. Yilbas and M. Kalyon, "Parametric variation of the maximum surface temperature during laser heating with convective boundary conditions," Journal of Mechanical Engineering Science, vol. 216, no. 6, pp. 691-699, 2002.

[7] M. A. Al-Nimr and V. S. Arpaci, "Picosecond thermal pulses in thin metal films," Journal of Applied Physics, vol. 85, no. 5, pp. 2517-2521, 1999.

[8] M. A. Al-Nimr, "Heat transfer mechanisms during short-duration laser heating of thin metal films," International Journal of Thermophysics, vol. 18, no. 5, pp. 1257-1268, 1997.

[9] T. Q. Qiu and C. L. Tien, "Short-pulse laser heating on metals," International Journal of Heat and Mass Transfer, vol. 35, no. 3, pp. 719-726, 1992.

[10] C. L. Tien and T. Q. Qiu, "Heat transfer mechanism during short pulse laser heating of metals," American Society of Mechanical Engineers Journal of Heat Transfer, vol. 115, pp. 835-841, 1993.

[11] C. Catteneo, "A form of heat conduction equation which eliminates the paradox of instantaneous propagation," Compte Rendus, vol. 247, pp. 431-433, 1958.

[12] P. Vernotte, "Some possible complications in the phenomenon of thermal conduction," Compte Rendus, vol. 252, pp. 2190-2191, 1961.

[13] M. A. Al-Nimr and V. S. Arpaci, "The thermal behavior of thin metal films in the hyperbolic two-step model," International Journal of Heat and Mass Transfer, vol. 43, no. 11, pp. 2021-2028, 2000.

[14] M. A. Al-Nimr, O. M. Haddad, and V. S. Arpaci, "Thermal behavior of metal films-A hyperbolic twostep model," Heat and Mass Transfer, vol. 35, no. 6, pp. 459-464, 1999.

[15] M. A. Al-Nimr, B. A. Abu-Hijleh, and M. A. Hader, "Effect of thermal losses on the microscopic hyperbolic heat conduction model," Heat and Mass Transfer, vol. 39, no. 3, pp. 201-207, 2003.

[16] M. Naji, M.A. Al-Nimr, and M. Hader, "The validity of using the microscopic hyperbolic heat conduction model under a harmonic fluctuating boundary heating source," International Journal of Thermophysics, vol. 24, no. 2, pp. 545-557, 2003.

[17] M. A. Al-Nimr and M. K. Alkam, "Overshooting phenomenon in the hyperbolic microscopic heat conduction model," International Journal of Thermophysics, vol. 24, no. 2, pp. 577-583, 2003.

[18] D. Y. Tzou, Macro-to-Microscale Heat Transfers-The Lagging Behavior, Taylor \& Francis, New York, NY, USA, 1997.

[19] C. I. Christov and P. M. Jordan, "Heat conduction paradox involving second-sound propagation in moving media," Physical Review Letters, vol. 94, no. 15, Article ID 154301, 4 pages, 2005.

[20] S. M. Zubair and M. A. Chaudhry, "Heat conduction in a semi-infinite solid due to time-dependent laser source," International Journal of Heat and Mass Transfer, vol. 39, no. 14, pp. 3067-3074, 1996.

[21] M. Lewandowska, "Hyperbolic heat conduction in the semi-infinite body with a time-dependent laser heat source," Heat and Mass Transfer, vol. 37, no. 4-5, pp. 333-342, 2001.

[22] S. H. Chan, J. D. Low, and W. K. Mueller, "Hyperbolic heat conduction in catalytic supported crystallites," AIChE Journal, vol. 17, pp. 1499-1501, 1971.

[23] L. G. Hector Jr., W. S. Kim, and M. N. Özisik, "Propagation and reflection of thermal waves in a finite medium due to axisymmetric surface sources," International Journal of Heat and Mass Transfer, vol. 35, no. 4, pp. 897-912, 1992.

[24] D. Y. Tzou, "The thermal shock phenomena induced by a rapidly propagating crack tip: experimental evidence," International Journal of Heat and Mass Transfer, vol. 35, no. 10, pp. 2347-2356, 1992.

[25] A. Vedavarz, S. Kumar, and M. K. Moallemi, "Significance of non-Fourier heat waves in conduction," Journal of Heat Transfer, vol. 116, no. 1, pp. 221-226, 1994.

[26] R. V. Churchill, Operational Mathematics, McGraw-Hill, New York, NY, USA, 1958. 


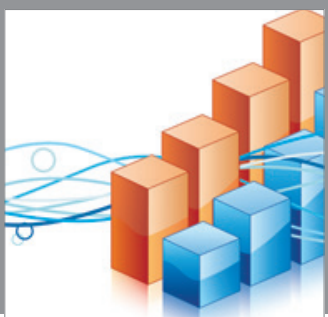

Advances in

Operations Research

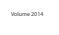

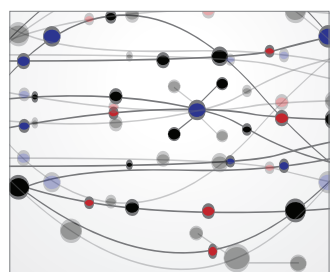

\section{The Scientific} World Journal
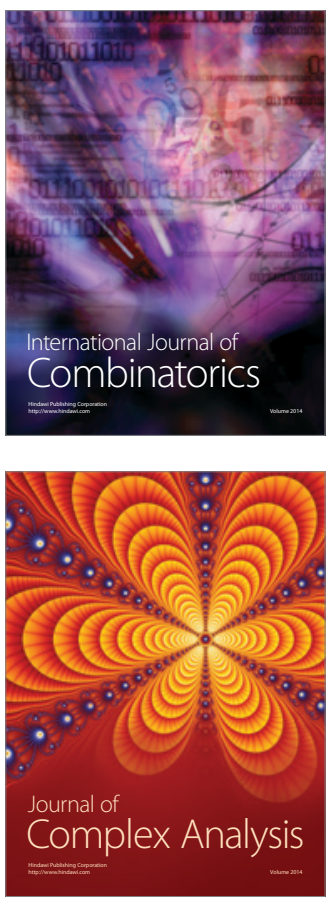

International Journal of

Mathematics and

Mathematical

Sciences
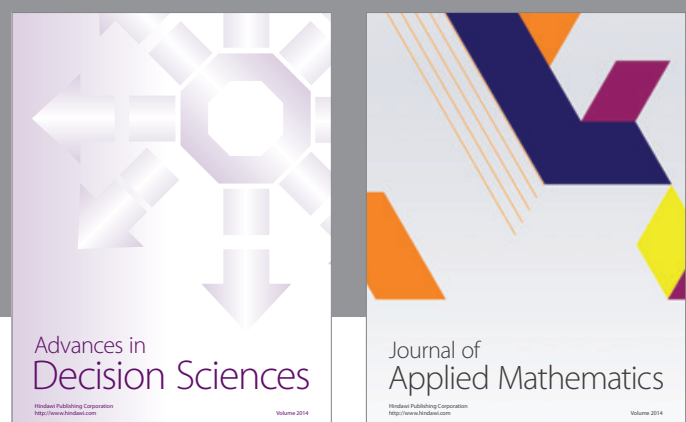

Journal of

Applied Mathematics
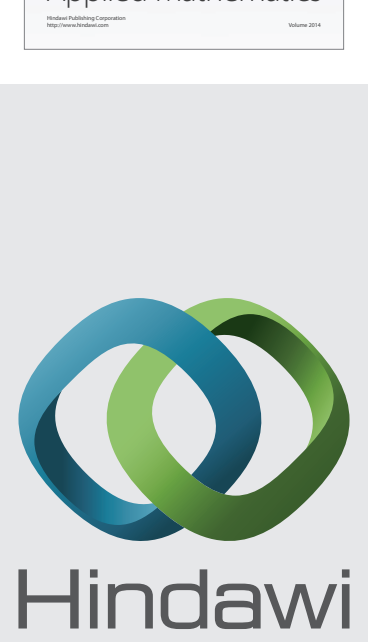

Submit your manuscripts at http://www.hindawi.com
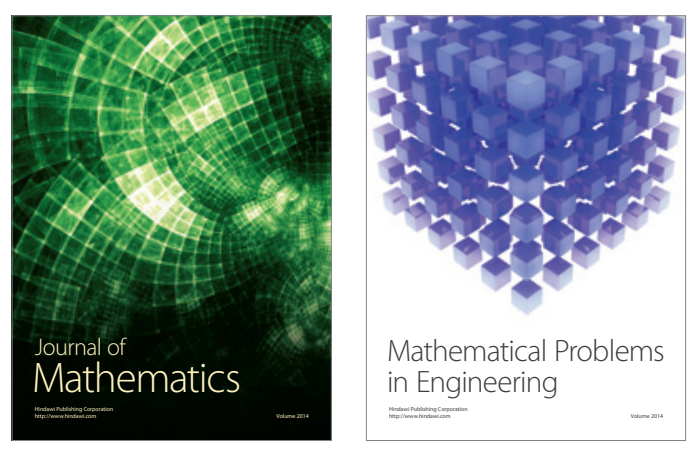

Mathematical Problems in Engineering
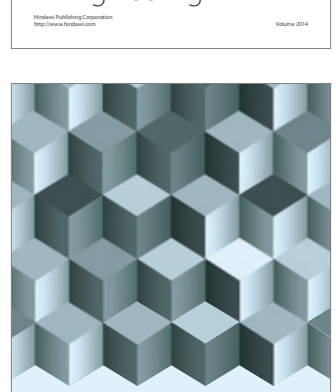

Journal of

Function Spaces
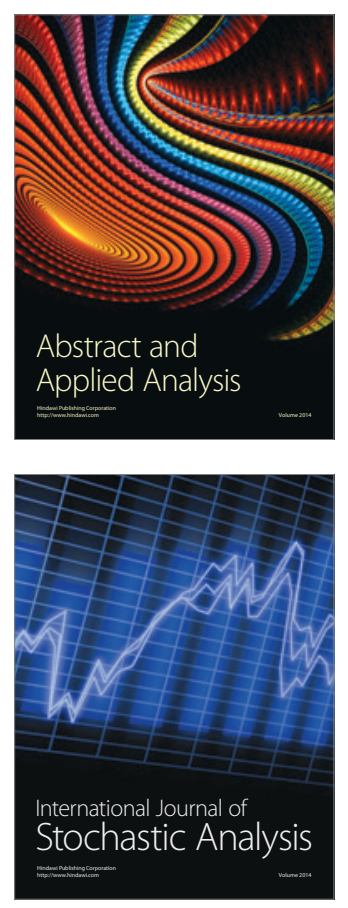

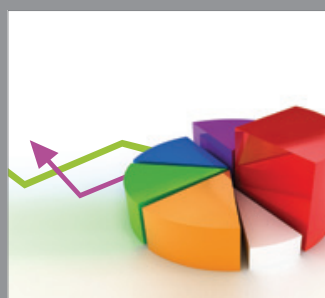

ournal of

Probability and Statistics

Promensencen
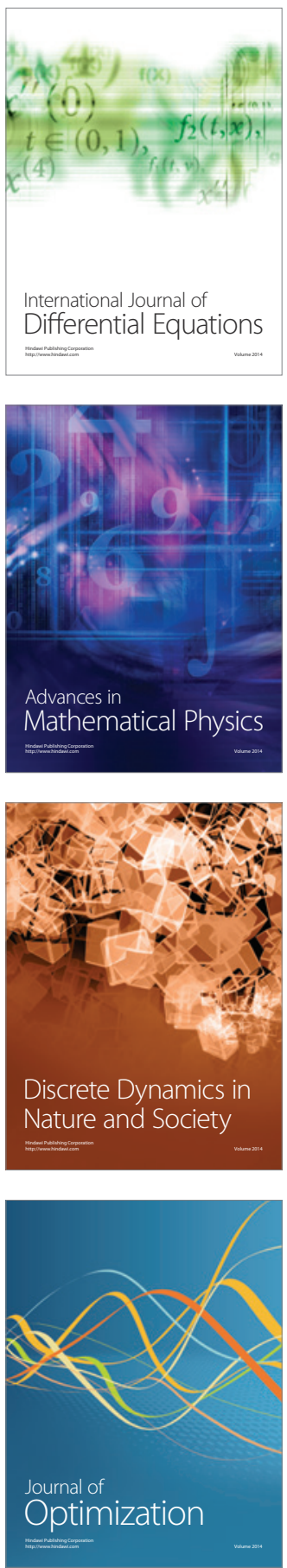